\title{
Efficiency of Ratio, Product, and Regression Estimators under Maximum and Minimum Values, Using Two Auxiliary Variables
}

\author{
Abdullah Y. Al-Hossain ${ }^{1}$ and Mursala Khan ${ }^{2}$ \\ ${ }^{1}$ Department of Mathematics, Faculty of Science, Jazan University, Jazan 2097, Saudi Arabia \\ ${ }^{2}$ Department of Mathematics, COMSATS Institute of Information Technology, Abbottabad, Pakistan
}

Correspondence should be addressed to Mursala Khan; mursala.khan@yahoo.com

Received 4 December 2013; Accepted 9 February 2014; Published 13 April 2014

Academic Editor: Bernard J. Geurts

Copyright ( 2014 A. Y. Al-Hossain and M. Khan. This is an open access article distributed under the Creative Commons Attribution License, which permits unrestricted use, distribution, and reproduction in any medium, provided the original work is properly cited.

\begin{abstract}
To obtain the best estimates of the unknown population parameters have been the key theme of the statisticians. In the present paper we have suggested some estimators which estimate the population parameters efficiently. In short we propose a ratio, product, and regression estimators using two auxiliary variables, when there are some maximum and minimum values of the study and auxiliary variables, respectively. The properties of the proposed strategies in terms of mean square errors (variances) are derived up to first order of approximation. Also the performance of the proposed estimators have shown theoretically and these theoretical conditions are verified numerically by taking four real data sets under which the proposed class of estimators performed better than the other previous works.
\end{abstract}

\section{Introduction}

In the literature of survey sampling, the use of ancillary information provided by auxiliary variables was discussed by various statisticians in order to improve the efficiency of their constructed estimators or to obtain improved estimators for estimating some most common population parameters, such as population mean, population total, population variance, and population coefficient of variation. In such a situation, ratio, product, and regression estimators provide better estimates of the population parameters. The work of Neyman [1] is considered as the early works where auxiliary information has been used. After that a lot of work has been done for estimating finite population mean and other population parameters using auxiliary information and for improving their efficiency. For a more related work one can go through Das and Tripathi [2,3], Upadhyaya and Singh [4], Singh [5], and so forth. Sisodia and Dwivedi [6] have proposed ratio estimator using coefficient of variation of an auxiliary variable. Kadilar and Cingi [7] have suggested an estimator for population mean using two auxiliary variables. Khan and Shabbir [8] have introduced the idea of ratio type estimator or the estimation of population variance using quartiles of an auxiliary variable. Mouatasim and Al-Hossain [9] have studied reduced gradient method for minimax estimation of a bounded poisson mean in which concept of auxiliary variables can be easily placed and study. Further Al-Hossain [10] has studied inference on compound Rayleigh parameters with progressively type II censored samples wherein censored samples can be chosen as to auxiliary variables. Recently Khan and Shabbir [11] suggested different estimators of finite population mean using maximum and minimum values.

Let us consider a finite population of size $N$ of different units $U=\left\{U_{1}, U_{2}, U_{3}, \ldots, U_{N}\right\}$. Let $y, x_{1}$, and $x_{2}$ be the study and the auxiliary variables with corresponding values $y_{i}, x_{1 i}$, and $x_{2 i}$, respectively, for the $i$ th unit $i=\{1,2,3, \ldots, N\}$ defined on a finite population $U$. Let $\bar{Y}=(1 / N) \sum_{i=1}^{N} y_{i}$, $\bar{X}_{1}=(1 / N) \sum_{i=1}^{N} x_{1 i}$, and $\bar{X}_{2}=(1 / N) \sum_{i=1}^{N} x_{2 i}$ be the population means of the study as well as auxiliary variables, respectively, let $S_{y}^{2}=(1 / N-1) \sum_{i=1}^{N}\left(y_{i}-\bar{Y}\right)^{2}, S_{x_{1}}^{2}=(1 / N-$ 1) $\sum_{i=1}^{N}\left(x_{1 i}-\bar{X}_{1}\right)^{2}$, and $S_{x_{2}}^{2}=(1 / N-1) \sum_{i=1}^{N}\left(x_{2 i}-\bar{X}_{2}\right)^{2}$ be the corresponding population variances of the study as well as auxiliary variables, respectively, let $C_{y}, C_{x_{1}}$, and $C_{x_{2}}$ be the coefficient of variation of the study as well as 
auxiliary variables, respectively, and let $\rho_{y x_{1}}, \rho_{y x_{2}}$, and $\rho_{x_{1} x_{2}}$ be the population correlation coefficient among $y, x_{1}, x_{2}$ and between $x_{1}$ and $x_{2}$, respectively.

In order to estimate the unknown population mean, we take a random sample of size $n$ units from the finite population $U$ by using simple random sample without replacement. Let $y, x_{1}$, and $x_{2}$ be the study and the auxiliary variables with corresponding values $y_{i}, x_{1 i}$, and $x_{2 i}$, respectively, for the $i$ th unit $i=\{1,2,3, \ldots, n\}$ in the sample. Let $\bar{y}=$ $(1 / n) \sum_{i=1}^{n} y_{i}, \bar{x}_{1}=(1 / n) \sum_{i=1}^{n} x_{1 i}$, and $\bar{x}_{2}=(1 / n) \sum_{i=1}^{n} x_{2 i}$ be the sample means of the study as well as auxiliary variables, respectively, and let $\widehat{S}_{y}^{2}=(1 / n-1) \sum_{i=1}^{n}\left(y_{i}-\bar{y}\right)^{2}, \widehat{S}_{x_{1}}^{2}=$ $(1 / n-1) \sum_{i=1}^{n}\left(x_{1 i}-\bar{x}_{1}\right)^{2}$, and $\widehat{S}_{x_{2}}^{2}=(1 / n-1) \sum_{i=1}^{n}\left(x_{2 i}-\bar{x}_{2}\right)^{2}$ be the corresponding sample variances of the study as well as auxiliary variables, respectively. Also let $\widehat{C}_{y}, \widehat{C}_{x_{1}}$, and $\widehat{C}_{x_{2}}$ be the sample coefficient of variation of the study variable $y$ as well as auxiliary variables $x_{1}$ and $x_{2}$, respectively, and let $\widehat{S}_{y x_{1}}$, $\widehat{S}_{y x_{2}}$, and $\widehat{S}_{x_{1} x_{2}}$ be the sample covariances between $y, x_{1}$, and $x_{2}$ and between $x_{1}$ and $x_{2}$, respectively.

The usual unbiased estimator to estimate the population mean of the study variable is

$$
\bar{y}=\frac{\sum_{i=1}^{n} y_{i}}{n}
$$

The variance of the estimator $\bar{y}$ up to first order of approximation is given as follows:

$$
\operatorname{var}(\bar{y})=\theta S_{y}^{2}
$$

where $\theta=1 / n-1 / N$.

In many real data sets there exist some large $\left(y_{\max }\right)$ or small values $\left(y_{\min }\right)$ and to estimate the unknown population parameters without considering this information is very sensitive in case the result will be either overestimated or underestimated. In order to handle this situation Sarndal [12] suggested the following unbiased estimator for the estimation of finite population mean using maximum and minimum values:

$$
\bar{y}_{S}= \begin{cases}\bar{y}+c & \text { if sample contains } y_{\min } \text { but not } y_{\max } \\ \bar{y}-c & \text { if sample contains } y_{\max } \text { but not } y_{\min } \\ \bar{y} & \text { for all other samples, }\end{cases}
$$

where $c$ is a constant, which is to be found for minimum variance.

The minimum variance of the estimator $\bar{y}_{S}$ up to first order of approximation is given as

$$
\operatorname{var}\left(\bar{y}_{S}\right)_{\min }=\operatorname{var}(\bar{y})-\frac{\theta\left(y_{\max }-y_{\min }\right)^{2}}{2(N-1)},
$$

where the optimum value of $c_{\mathrm{opt}}$ is

$$
c_{\mathrm{opt}}=\frac{\left(y_{\max }-y_{\min }\right)}{2 n} \text {. }
$$

The ratio estimator for estimating the unknown population mean of the study variable using two auxiliary variables is given by

$$
\widehat{\bar{Y}}_{R 2}=\bar{y} \frac{\bar{X}_{1} \bar{X}_{2}}{\bar{x}_{1} \bar{x}_{2}} \text {. }
$$

The mean square error of the estimator $\widehat{\bar{Y}}_{R 2}$ up to first order of approximation is given by

$$
\begin{gathered}
\operatorname{MSE}\left(\hat{\bar{Y}}_{R 2}\right)=\theta\left[S_{y}^{2}+R_{1}^{2} S_{x_{1}}^{2}+R_{2}^{2} S_{x_{2}}^{2}+2 R_{1} R_{2} S_{x_{1} x_{2}}\right. \\
\left.-2 R_{2} S_{y x_{2}}-2 R_{1} S_{y x_{1}}\right] .
\end{gathered}
$$

The product estimator for estimating the unknown population mean of the study variable using two auxiliary variables is given by

$$
\widehat{\bar{Y}}_{P 2}=\bar{y} \frac{\bar{x}_{1} \bar{x}_{2}}{\bar{X}_{1} \bar{X}_{2}}
$$

The mean square error of the estimator $\widehat{\bar{Y}}_{P 2}$ up to first order of approximation is given by

$$
\begin{aligned}
\operatorname{MSE}\left(\hat{\bar{Y}}_{P 2}\right)=\theta\left[S_{y}^{2}\right. & +R_{1}^{2} S_{x_{1}}^{2}+R_{2}^{2} S_{x_{2}}^{2}+2 R_{1} R_{2} S_{x_{1} x_{2}} \\
& \left.+2 R_{2} S_{y x_{2}}+2 R_{1} S_{y x_{1}}\right] .
\end{aligned}
$$

When there are two auxiliary variables, then the regression estimator to estimate the finite population mean is given by

$$
\widehat{\bar{Y}}_{l r 2}=\bar{y}+b_{1}\left(\bar{X}_{1}-\bar{x}_{1}\right)+b_{2}\left(\bar{X}_{2}-\bar{x}_{2}\right),
$$

where $b_{1}=S_{y x_{1}} / S_{x_{1}}^{2}$ and $b_{2}=S_{y x_{2}} / S_{x_{2}}^{2}$ are the sample regression coefficients between $y$ and $x_{1}$ and between $y$ and $x_{2}$, respectively.

The variance of the estimator $\widehat{\bar{Y}}_{l r 2}$ up to first order of approximation is given as

$$
\operatorname{MSE}\left(\widehat{\bar{Y}}_{l r 2}\right)=\theta S_{y}^{2}\left[1-\rho_{y x_{1}}^{2}-\rho_{y x_{2}}^{2}+2 \rho_{y x_{1}} \rho_{y x_{2}} \rho_{x_{1} x_{2}}\right] .
$$

\section{Proposed Estimators}

On the lines of Sarndal [12], we propose a ratio, product, and regression estimators using two auxiliary variables when there are some maximum and minimum values of the study variables and the auxiliary variables, respectively.

Case 1. When the correlation between the study variable and the auxiliary variable is positive, the selection of the larger value of the auxiliary variable the larger the value of study variable is to be expected, and the smaller the value of auxiliary variable the smaller the value of study variable is 
to be expected, and using such type of information the ratio estimator using two auxiliary variables becomes

$$
\begin{aligned}
\hat{\bar{Y}}_{R C 2}= & \bar{y}_{C_{11}} \frac{\bar{X}_{1}}{\bar{x}_{1 C_{21}}} \frac{\bar{X}_{2}}{\bar{x}_{2 C_{31}}} \\
= & \left\{\begin{array}{l}
\left(\bar{y}+c_{1}\right) \frac{\bar{X}_{1}}{\left(\bar{x}_{1}+c_{2}\right)} \frac{\bar{X}_{2}}{\left(\bar{x}_{2}+c_{3}\right)} \\
\text { if sample contains } y_{\min } \text { and }\left(x_{1 \min }, x_{2 \min }\right), \\
\left(\bar{y}-c_{1}\right) \frac{\bar{X}_{1}}{\left(\bar{x}_{1}-c_{2}\right)} \frac{\bar{X}_{2}}{\left(\bar{x}_{2}-c_{3}\right)} \\
\text { if sample contains } y_{\max } \text { and }\left(x_{1 \max }, x_{2 \max }\right), \\
\bar{X}_{1} \bar{X}_{2} \bar{x}_{1} \bar{x}_{2} \\
\hat{\bar{Y}}_{\operatorname{lr}(P 1)}=\bar{y}_{C_{11}}+b_{1}\left(\bar{X}_{1}-\bar{x}_{1 C_{21}}\right)+b_{2}\left(\bar{X}_{2}-\bar{x}_{2 C_{31}}\right)
\end{array}\right.
\end{aligned}
$$

where $\left(\bar{y}_{C_{11}}=\bar{y}+c_{1}, \bar{x}_{1 C_{21}}=\bar{x}_{1}+c_{2}, \bar{x}_{2 C_{31}}=\bar{x}_{2}+c_{3}\right)$ if the sample contains $y_{\min }$ and $\left(x_{1 \mathrm{~min}}, x_{2 \mathrm{~min}}\right) \cdot\left(\bar{y}_{C_{12}}=\bar{y}-c_{1}\right.$, $\left.\bar{x}_{2 C_{22}}=\bar{x}_{1}-c_{2}, \bar{x}_{2 C_{32}}=\bar{x}_{2}-c_{3}\right)$ if the sample contains $y_{\max }$ and $\left(x_{1 \max }, x_{2 \max }\right)$. And $\left(\bar{y}_{C_{11}}=\bar{y}, \bar{x}_{1 C_{21}}=\bar{x}_{1}, \bar{x}_{2 C_{31}}=\bar{x}_{2}\right)$ for all other samples.

Case 2. Similarly when the correlation is negative the selection of the larger value of the auxiliary variable the smaller the value of study variable is to be expected, and the smaller the value of auxiliary variable the larger the value of study variable is to be expected, and using such type of information the product estimator using two auxiliary variables becomes

$$
\begin{aligned}
\hat{\bar{Y}}_{P C 2}= & \bar{y}_{C_{12}} \frac{\bar{x}_{1 C_{22}} \frac{\bar{x}_{2 C_{32}}}{\bar{X}_{1}}}{\bar{X}_{2}} \\
= & \left\{\begin{array}{c}
\left(\bar{y}+c_{1}\right) \frac{\left(\bar{x}_{1}-c_{2}\right)}{\bar{X}_{1}} \frac{\left(\bar{x}_{2}-c_{3}\right)}{\bar{X}_{2}} \\
\quad \text { if sample contains } y_{\text {min }} \text { sand }\left(x_{1 \max }, x_{2 \max }\right), \\
\left(\bar{y}-c_{1}\right) \frac{\left(\bar{x}_{1}+c_{2}\right)}{\bar{X}_{1}} \frac{\left(\bar{x}_{2}+c_{3}\right)}{\bar{X}_{2}} \\
\quad \text { if sample contains } y_{\max } \text { and }\left(x_{1 \min }, x_{2 \min }\right), \\
\bar{x}_{1} \bar{x}_{2} \text { for all other samples, } \\
\hat{\bar{X}}_{1} \bar{X}_{\operatorname{lr}(P 2)}=\bar{y}_{C_{12}}+b_{1}\left(\bar{X}_{1}-\bar{x}_{1 C_{22}}\right)+b_{2}\left(\bar{X}_{2}-\bar{x}_{2 C_{32}}\right),
\end{array}\right.
\end{aligned}
$$

where $\left(\bar{y}_{C_{12}}=\bar{y}+c_{1}, \bar{x}_{1 C_{22}}=\bar{x}_{1}-c_{2}, \bar{x}_{2 C_{32}}=\bar{x}_{2}-c_{3}\right)$ if the sample contains $y_{\min }$ and $\left(x_{1 \max }, x_{2 \max }\right),\left(\bar{y}_{C_{11}}=\bar{y}-c_{1}\right.$, $\left.\bar{x}_{1 C_{21}}=\bar{x}_{1}+c_{2}, \bar{x}_{2 C_{31}}=\bar{x}_{2}+c_{3}\right)$ if the sample contains $y_{\max }$ and $\left(x_{1 \mathrm{~min}}, x_{2 \mathrm{~min}}\right)$, and $\left(\bar{y}_{C_{12}}=\bar{y}, \bar{x}_{1 C_{22}}=\bar{x}_{1}, \bar{x}_{2 C_{32}}=\bar{x}_{2}\right)$ for all other samples. Also $c_{1}, c_{2}$, and $c_{3}$ are unknown constants, whose value is to be determined for optimality conditions.

To obtain the properties of the proposed estimators in terms of bias and mean square error, we define the following relative error terms and their expectations.
$e_{0}=\left(\bar{y}_{c_{1}}-\bar{Y}\right) / \bar{Y}, e_{1}=\left(\bar{x}_{1 c_{2}}-\bar{X}_{1}\right) / \bar{X}_{1}$, and $e_{2}=\left(\bar{x}_{2 c_{3}}-\right.$ $\left.\bar{X}_{2}\right) / \bar{X}_{2}$, such that $E\left(e_{0}\right)=E\left(e_{1}\right)=E\left(e_{2}\right)=0$. Consider also

$$
\begin{aligned}
& E\left(e_{0}^{2}\right)=\frac{\theta}{\bar{Y}^{2}}\left(s_{y}^{2}-\frac{2 n c_{1}}{N-1}\left(y_{\max }-y_{\min }-n c_{1}\right)\right), \\
& E\left(e_{1}^{2}\right)=\frac{\theta}{\bar{X}_{1}^{2}}\left(S_{x_{1}}^{2}-\frac{2 n c_{2}}{N-1}\left(x_{1 \max }-x_{1 \min }-n c_{2}\right)\right), \\
& E\left(e_{2}^{2}\right)=\frac{\theta}{\bar{X}_{2}^{2}}\left(S_{x_{2}}^{2}-\frac{2 n c_{3}}{N-1}\left(x_{2 \max }-x_{2 \min }-n c_{3}\right)\right) \\
& E\left(e_{0} e_{1}\right)=\frac{\theta}{\overline{Y X}_{1}}\left(s_{y x_{1}}-\frac{n}{N-1}\right. \\
& \times\left(c_{2}\left(y_{\max }-y_{\min }\right)\right. \\
& \left.\left.+c_{1}\left(x_{1 \max }-x_{1 \min }\right)-2 n c_{1} c_{2}\right)\right), \\
& E\left(e_{0} e_{2}\right)=\frac{\theta}{\overline{Y X}_{2}}\left(s_{y x_{2}}-\frac{n}{N-1}\right. \\
& \times\left(c_{3}\left(y_{\max }-y_{\min }\right)\right. \\
& \left.\left.+c_{1}\left(x_{2 \max }-x_{2 \min }\right)-2 n c_{1} c_{3}\right)\right), \\
& E\left(e_{1} e_{2}\right)=\frac{\theta}{\bar{X}_{1} \bar{X}_{2}}\left(S_{x_{1} x_{2}}-\frac{n}{N-1}\right. \\
& \times\left(c_{3}\left(x_{1 \max }-x_{1 \min }\right)\right. \\
& \left.\left.+c_{2}\left(x_{2 \max }-x_{2 \min }\right)-2 n c_{2} c_{3}\right)\right) \text {. }
\end{aligned}
$$

Rewriting (12), $\widehat{\bar{Y}}_{R C 2}$ in terms of $e_{i}$ 's, we have

$$
\widehat{\bar{Y}}_{R C 2}=\bar{Y}\left(1+e_{0}\right)\left(1+e_{1}\right)^{-1}\left(1+e_{2}\right)^{-1} .
$$

Expanding the right hand side of above equation and including terms up to second powers of $e_{i}$ 's, that is, up to first order of approximation, we have

$$
\widehat{\bar{Y}}_{R C 2}-\bar{Y}=\bar{Y}\left(e_{0}-e_{1}-e_{2}+e_{2}^{2}+e_{1}^{2}+e_{1} e_{2}-e_{0} e_{2}-e_{0} e_{1}\right) .
$$

On squaring both sides of (17) and keeping $e_{i}$ 's powers up to first order of approximation, we have

$$
\left(\widehat{\bar{Y}}_{R C 2}-\bar{Y}\right)^{2}=\bar{Y}^{2}\left[e_{0}^{2}+e_{1}^{2}+e_{2}^{2}-2 e_{0} e_{1}-2 e_{0} e_{2}+2 e_{1} e_{2}\right]
$$


Taking expectation on both sides of (18), we get mean square error up to first order of approximation, given as

$$
\begin{aligned}
& \operatorname{MSE}\left(\hat{\bar{Y}}_{R C 2}\right) \\
& =\left[\theta \left(S_{y}^{2}+R_{1}^{2} S_{x_{1}}^{2}+R_{2}^{2} S_{x_{2}}^{2}+2 R_{1} R_{2} S_{x_{1} x_{2}}\right.\right. \\
& \left.\quad-2 R_{2} S_{y x_{2}}-2 R_{1} S_{y x_{1}}\right)-\frac{2 n \theta\left(c_{1}-R_{2} c_{3}-R_{1} c_{2}\right)}{N-1} \\
& \quad \times\left\{\left(y_{\text {max }}-y_{\text {min }}\right)-R_{1}\left(x_{1 \max }-x_{1 \min }\right)\right. \\
& \left.\left.\quad-R_{2}\left(x_{2 \max }-x_{2 \min }\right)-n\left(c_{1}-R_{2} c_{3}-R_{1} c_{2}\right)\right\}\right] .
\end{aligned}
$$

To find the minimum mean squared error of $\hat{\bar{Y}}_{R C 2}$, we differentiate (19) with respect to $c_{1}, c_{2}$, and $c_{3}$, respectively; that is,

$$
\begin{gathered}
\frac{\partial \operatorname{MSE}\left(\hat{\bar{Y}}_{R C 2}\right)}{\partial c_{1}}=0 \text { or } \\
\left(y_{\text {max }}-y_{\text {min }}\right)-R_{1}\left(x_{1 \text { max }}-x_{1 \text { min }}\right) \\
-R_{2}\left(x_{2 \text { max }}-x_{2 \text { min }}\right)-2 n\left(c_{1}-R_{1} c_{2}-R_{2} c_{3}\right)=0, \\
\frac{\partial \operatorname{MSE}\left(\hat{\bar{Y}}_{R C 2}\right)}{\partial c_{2}}=0 \quad \text { or } \\
\left(y_{\text {max }}-y_{\text {min }}\right)-R_{1}\left(x_{1 \max }-x_{1 \text { min }}\right) \\
-R_{2}\left(x_{2 \max }-x_{2 \min }\right)-2 n\left(c_{1}-R_{1} c_{2}-R_{2} c_{3}\right)=0, \\
\frac{\partial \mathrm{MSE}\left(\hat{\bar{Y}}_{R C 2}\right)}{\partial c_{3}}=0 \quad \text { or } \\
\left(y_{\text {max }}-y_{\text {min }}\right)-R_{1}\left(x_{1 \max }-x_{1 \min }\right) \\
-R_{2}\left(x_{2 \max }-x_{2 \min }\right)-2 n\left(c_{1}-R_{1} c_{2}-R_{2} c_{3}\right)=0 .
\end{gathered}
$$

On differentiating (19), with respect to $c_{1}, c_{2}$, and $c_{3}$, respectively, we get one equation with three unknowns and so unique solution is not possible; so let

$$
\begin{aligned}
& c_{1}=\frac{\left(y_{\max }-y_{\min }\right)}{2 n}, \\
& c_{2}=\frac{\left(x_{1 \mathrm{max}}-x_{1 \mathrm{~min}}\right)}{2 n}, \\
& c_{3}=\frac{\left(x_{2 \max }-x_{2 \mathrm{~min}}\right)}{2 n} .
\end{aligned}
$$

On substituting the optimum value of $c_{1}, c_{2}$, and $c_{3}$ from (21) in (19), we get the minimum mean square error of the proposed estimator, given as

$$
\begin{aligned}
\operatorname{MSE}\left(\hat{\bar{Y}}_{R C 2}\right)_{\min } \\
=\operatorname{MSE}\left(\hat{\bar{y}}_{R 2}\right)-\frac{\theta}{2(N-1)} \\
\quad \times\left[\left(y_{\text {max }}-y_{\text {min }}\right)\right. \\
\left.\quad-R_{1}\left(x_{1 \text { max }}-x_{1 \text { min }}\right)-R_{2}\left(x_{2 \text { max }}-x_{2 \text { min }}\right)\right]^{2},
\end{aligned}
$$

where $\operatorname{MSE}\left(\hat{\bar{Y}}_{R 2}\right)=\theta\left[S_{y}^{2}+R_{1}^{2} S_{x_{1}}^{2}+R_{2}^{2} S_{x_{2}}^{2}+2 R_{1} R_{2} S_{x_{1} x_{2}}-\right.$ $\left.2 R_{2} S_{y x_{2}}-2 R_{1} S_{y x_{1}}\right]$.

Similarly the mean square error of the product estimator, up to first order of approximation, is given by

$$
\begin{aligned}
\operatorname{MSE}\left(\hat{\bar{Y}}_{P C 2}\right)_{\min } \\
=\operatorname{MSE}\left(\hat{\bar{y}}_{P 2}\right)-\frac{\theta}{2(N-1)} \\
\quad \times\left[\left(y_{\text {max }}-y_{\text {min }}\right)\right. \\
\left.\quad+R_{1}\left(x_{1 \text { max }}-x_{1 \text { min }}\right)+R_{2}\left(x_{2 \text { max }}-x_{2 \text { min }}\right)\right]^{2},
\end{aligned}
$$

where $\operatorname{MSE}\left(\widehat{\bar{Y}}_{P 2}\right)=\theta\left[S_{y}^{2}+R_{1}^{2} S_{x_{1}}^{2}+R_{2}^{2} S_{x_{2}}^{2}+2 R_{1} R_{2} S_{x_{1} x_{2}}+\right.$ $\left.2 R_{2} S_{y x_{2}}+2 R_{1} S_{y x_{1}}\right]$.

Now the minimum variance of the regression estimator in the case of positive correlation, up to first order of approximation, is given by

$$
\begin{aligned}
\operatorname{MSE}\left(\hat{\bar{Y}}_{l r(P 1)}\right)_{\min } \\
=\operatorname{MSE}\left(\widehat{\bar{Y}}_{l r 2}\right)-\frac{\theta}{2(N-1)} \\
\quad \times\left(\left(y_{\max }-y_{\text {min }}\right)\right. \\
\left.\quad-\beta_{1}\left(x_{1 \text { max }}-x_{1 \text { min }}\right)-\beta_{2}\left(x_{2 \text { max }}-x_{2 \min }\right)\right)^{2},
\end{aligned}
$$

where $\operatorname{MSE}\left(\widehat{\bar{Y}}_{l r 2}\right)=\theta S_{y}^{2}\left[1-\rho_{y x_{1}}^{2}-\rho_{y x_{2}}^{2}+2 \rho_{y x_{1}} \rho_{y x_{2}} \rho_{x_{1} x_{2}}\right]$.

Similarly for the case of negative correlation, the minimum variance of the regression estimator, up to first order of approximation, is given by

$$
\begin{aligned}
& \operatorname{MSE}\left(\hat{\bar{Y}}_{l r(P 2)}\right)_{\min } \\
& =\operatorname{MSE}\left(\hat{\bar{Y}}_{l r 2}\right)-\frac{\theta}{2(N-1)} \\
& \quad \times\left[\left(y_{\max }-y_{\text {min }}\right)\right. \\
& \left.\quad+\beta_{1}\left(x_{1 \text { max }}-x_{1 \text { min }}\right)+\beta_{2}\left(x_{2 \text { max }}-x_{2 \text { min }}\right)\right]^{2} .
\end{aligned}
$$


But when there is positive and negative correlation, the regression estimator gives us better result, and so for both cases (positive and negative correlation) we write the variance as

$$
\begin{aligned}
\operatorname{MSE}\left(\hat{\bar{Y}}_{\operatorname{lr}(P)}\right)_{\min } \\
=\operatorname{MSE}\left(\hat{\bar{Y}}_{l r 2}\right)-\frac{\theta}{2(N-1)} \\
\quad \times\left(\left(y_{\max }-y_{\text {min }}\right)\right. \\
\left.\quad-\left|\beta_{1}\right|\left(x_{1 \text { max }}-x_{1 \text { min }}\right)-\left|\beta_{2}\right|\left(x_{2 \text { max }}-x_{2 \text { min }}\right)\right)^{2} .
\end{aligned}
$$

\section{Comparison of Estimators}

In this section, we have compared the proposed estimators with the ratio, product, and regression estimators and some of their efficiency comparison condition has been carried out under which the proposed estimators perform better.

(i) By (7) and (22),

$$
\left[\operatorname{MSE}\left(\widehat{\bar{Y}}_{R 2}\right)-\operatorname{MSE}\left(\widehat{\bar{Y}}_{R C 2}\right)_{\min }\right] \geq 0
$$

if

$$
\begin{aligned}
& {\left[\left(y_{\max }-y_{\min }\right)-R_{1}\left(x_{1 \max }-x_{1 \min }\right)\right.} \\
& \left.-R_{2}\left(x_{2 \max }-x_{2 \min }\right)\right]^{2} \geq 0 .
\end{aligned}
$$

(ii) By (9) and (23),

$$
\left[\operatorname{MSE}\left(\widehat{\bar{Y}}_{P 2}\right)-\operatorname{MSE}\left(\widehat{\bar{Y}}_{P C 2}\right)_{\min }\right] \geq 0
$$

if

$$
\begin{aligned}
& {\left[\left(y_{\max }-y_{\min }\right)+R_{1}\left(x_{1 \max }-x_{1 \min }\right)\right.} \\
& \left.+R_{2}\left(x_{2 \max }-x_{2 \min }\right)\right]^{2} \geq 0 .
\end{aligned}
$$

(iii) By (11) and (26),

$$
\left[\operatorname{MSE}\left(\widehat{\bar{Y}}_{l r 2}\right)-\operatorname{MSE}\left(\hat{\bar{Y}}_{l r(P)}\right)_{\min }\right] \geq 0
$$

if

$$
\begin{gathered}
{\left[\left(y_{\max }-y_{\min }\right)-\left|\beta_{1}\right|\left(x_{1 \max }-x_{1 \min }\right)\right.} \\
\left.-\left|\beta_{2}\right|\left(x_{2 \max }-x_{2 \min }\right)\right]^{2} \geq 0 .
\end{gathered}
$$

From (i), (ii), and (iii) we have observed that the proposed estimators performed better than the other existing estimators because the conditions are in the form of a square and greater than zero which is always true.

\section{Numerical Illustration}

In this section we demonstrate the performance of the suggested estimators over various other estimators, through four real data sets. The description and the necessary data statistics of the populations are given as follows.

Population 1 (source: Agricultural Statistics (1999) [13], Washington, DC.)

$Y$ : estimated number of fish caught during 1995;

$X_{1}$ : estimated number of fish caught during 1994;

$X_{2}$ : estimated number of fish caught during 1993;

$N=69, n=20, \bar{X}_{1}=4954.435, \bar{X}_{2}=4591.072$,

$\bar{Y}=4514.899, S_{y}^{2}=37199578, S_{x_{2}}^{2}=39881874, C_{y}^{2}=$ $1.8249, C_{x_{1}}^{2}=2.0300, C_{x_{2}}^{2}=1.8921, x_{1 \max }=38007$, $x_{1 \text { min }}=32, y_{\text {max }}=30027, y_{\text {min }}=23, x_{2 \max }=34060$, $x_{2 \min }=35, S_{x_{1}}^{2}=49829270, \rho_{y x_{1}}=0.9601, \rho_{y x_{2}}=$ $0.9564, \rho_{x_{1} x_{2}}=0.9729, S_{y x_{1}}=41335932.85$, and $S_{y x_{2}}=$ 36838026.14 .

Population 2 (source: Agricultural Statistics (1998) [14], Washington, US.)

$Y$ : season average price per pound during 1996;

$X_{1}$ : season average price per pound during 1995;

$X_{2}$ : season average price per pound during 1994;

$N=36, n=12, \bar{X}_{1}=0.1856, \bar{X}_{2}=0.1708$, $\bar{Y}=0.2033, x_{1 \max }=0.403, x_{1 \min }=0.071, y_{\max }=$ $0.452, y_{\min }=0.101, x_{2 \max }=0.334, x_{2 \min }=0.078$, $S_{y}^{2}=0.006458, S_{x_{1}}^{2}=0.005654, S_{x_{2}}^{2}=0.004017, C_{y}^{2}=$ $0.1563, C_{x_{1}}^{2}=0.1641, C_{x_{2}}^{2}=0.13757, \rho_{y x_{1}}=0.8775$, $\rho_{y x_{2}}=0.8577, \rho_{x_{1} x_{2}}=0.8788, S_{y x_{1}}=0.0053$, and $S_{y x_{2}}=0.0044$.

Population 3 (source: Agricultural Statistics (1999) [13], Washington, DC.)

$Y$ : estimated number of fish caught during 1995;

$X_{1}$ : estimated number of fish caught during 1994;

$X_{2}$ : estimated number of fish caught during 1992;

$N=69, n=20, \bar{X}_{1}=4954.435, \bar{X}_{2}=4230.174, \bar{Y}=$ $4514.899, x_{1 \max }=38007, x_{1 \min }=32, S_{y}^{2}=37199578$, $y_{\max }=30027, y_{\min }=23, S_{x_{1}}^{2}=49829270, S_{x_{2}}^{2}=$ $31010599, x_{2 \max }=38933, x_{2 \min }=5, C_{y}^{2}=1.8249$, $C_{x_{1}}^{2}=2.0300, C_{x_{2}}^{2}=1.7329, S_{y x_{1}}=41335932.85$, $S_{y x_{2}}=32395255.07, S_{x_{1} x_{2}}=37642761.85, \rho_{y x_{1}}=$ $0.9601, \rho_{y x_{2}}=0.9538$, and $\rho_{x_{1} x_{2}}=0.9576$.

Population 4 (source: Agricultural Statistics (1999) [13], Washington, DC.)

$Y$ : estimated number of fish caught during 1995; $X_{1}$ : estimated number of fish caught during 1993; 
TABLE 1: MSE of the existing and the proposed estimators.

\begin{tabular}{lcccc}
\hline Estimator & $\begin{array}{c}\text { Population 1 } \\
\text { MSE }(\cdot)\end{array}$ & $\begin{array}{c}\text { Population } 2 \\
\text { MSE }(\cdot)\end{array}$ & $\begin{array}{c}\text { Population 3 } \\
\text { MSE }(\cdot)\end{array}$ & $\begin{array}{c}\text { Population } 4 \\
\text { MSE }(\cdot)\end{array}$ \\
\hline$\hat{\bar{Y}}_{R 2}$ & 1671738.947 & 0.0004 & 1513620.253 & 1440668.498 \\
$\widehat{\bar{Y}}_{P 2}$ & 12164963.92 & 0.0029 & 11772394.82 & 11494548.32 \\
$\widehat{\bar{Y}}_{l r 2}$ & 1254819.885 & 0.0003 & 1217975.563 & 1231973.764 \\
Proposed & & & & \\
$\quad \widehat{\bar{Y}}_{R C 2}$ & 1293565.557 & 0.0003 & 957662.57 & \\
$\widehat{\bar{Y}}_{P C 2}$ & 9654413.760 & 0.0021 & 8830692.238 & \\
$\widehat{\widehat{Y}}_{l r P}$ & 971781.5401 & 0.00025 & 753868.3966 & \\
\hline
\end{tabular}

$X_{2}$ : estimated number of fish caught during 1992;

$N=69, n=20, \bar{X}_{1}=4591.072, \bar{X}_{2}=4230.174, \bar{Y}=$ 4514.899, $y_{\max }=30027, y_{\min }=23, x_{1 \max }=34060$, $x_{1 \mathrm{~min}}=35, S_{y}^{2}=37199578, S_{x_{1}}^{2}=39881874, S_{x_{2}}^{2}=$ $31010599, C_{y}^{2}=1.8249, x_{2 \max }=28933, x_{2 \min }=5$, $C_{x_{1}}^{2}=1.8921, C_{x_{2}}^{2}=1.7329, S_{y x_{1}}=36838026.14, \rho_{y x_{1}}=$ $0.9564, S_{x_{1} x_{2}}=33873441.88, S_{y x_{2}}=32395255.07$, $\rho_{y x_{2}}=0.9538$, and $\rho_{x_{1} x_{2}}=0.9632$.

The mean squared error of the proposed and the existing estimators is shown in Table 1.

\section{Conclusion and Future Work}

We have developed some ratio, product, and regression estimators under maximum and minimum values using two auxiliary variables. The proposed estimators under certain efficiency conditions are shown to be more efficient than the ratio, product, and regression estimators using two auxiliary variables. The results are shown numerically in Table 1 where we observed that the performance of the proposed estimators is better than the usual ratio, product, and the regression estimators using two auxiliary variables. We can easily implement the concept of auxiliary variables minimax or maximin estimation of a bounded Poisson (respectively some other distribution) mean and censors samples. Thus the proposed estimators may be preferred over the existing estimators for the use of practical applications.

\section{Conflict of Interests}

The authors declare that there is no conflict of interests regarding the publication of this paper.

\section{References}

[1] J. Neyman, "Contributions to the theory of sampling human populations," Journal of the American Statistical Association, vol. 33, no. 201, pp. 101-116, 1938.

[2] A. K. Das and T. P. Tripathi, "Sampling strategies for population mean when the coefficient of variation of an auxiliary character is known," Sankhya, vol. 42, pp. 76-86, 1980.

[3] A. K. Das and T. P. Tripathi, "A class of sampling strategies for population mean using information on mean and variance of an auxiliary character," in Proceedings of the Indian Statistical Institute Golden Jubilee International Conference on Statistics: Applications and New Directions, pp. 174-181, Calcutta, India, December 1981.

[4] L. N. Upadhyaya and H. P. Singh, "Use of transformed auxiliary variable in estimating the finite population mean," Biometrical Journal, vol. 41, no. 5, pp. 627-636, 1999.

[5] S. Singh, "Golden and Silver Jubilee year-2003 of the linear regression estimators," in Proceedings of the American Statistical Association, Survey Method Section, pp. 4382-4389, American Statistical Association, Toronto, Canada, 2004.

[6] B. V. S. Sisodia and V. K. Dwivedi, "A modified ratio estimator using coefficient of variation of auxiliary variable," Journal of the Indian Society of Agricultural Statistics, vol. 33, no. 1, pp. 13-18, 1981.

[7] C. Kadilar and H. Cingi, "A new estimator using two auxiliary variables," Applied Mathematics and Computation, vol. 162, no. 2, pp. 901-908, 2005.

[8] M. Khan and J. Shabbir, "A ratio type estimator for the estimation of population variance using quartiles of an auxiliary variable," Journal of Statistics Applications and Probability, vol. 2, no. 3, pp. 157-162, 2013.

[9] A. E. Mouatasim and A. Al-Hossain, "Reduced gradient method for minimax estimation of a bounded poisson mean," Journal of Statistics: Advances in Theory and Applications, vol. 2, no. 2, pp. 185-199, 2009.

[10] Y. Al-Hossain, "Inferences on compound rayleigh parameters with progressively type-II censored samples," World Academy of Science, Engineering and Technology, vol. 76, pp. 885-892, 2013.

[11] M. Khan and J. Shabbir, "Some improved ratio, product and regression estimators of finite population mean when using minimum and maximum values," The Scientific World Journal, vol. 2013, Article ID 431868, 7 pages, 2013.

[12] C. E. Sarndal, "Sample survey theory vs general statistical theory: estimation of the population mean," International Statistical Institute, vol. 40, pp. 1-12, 1972.

[13] http://www.nass.usda.gov/Publications/Ag_Statistics/1999/ index.asp.

[14] http://www.nass.usda.gov/Publications/Ag_Statistics/. 


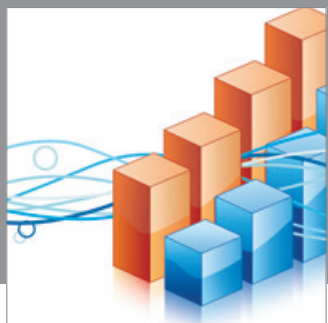

Advances in

Operations Research

mansans

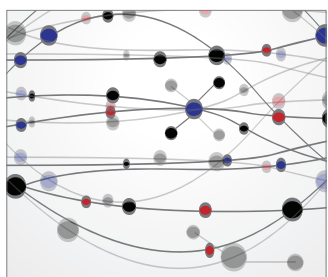

The Scientific World Journal
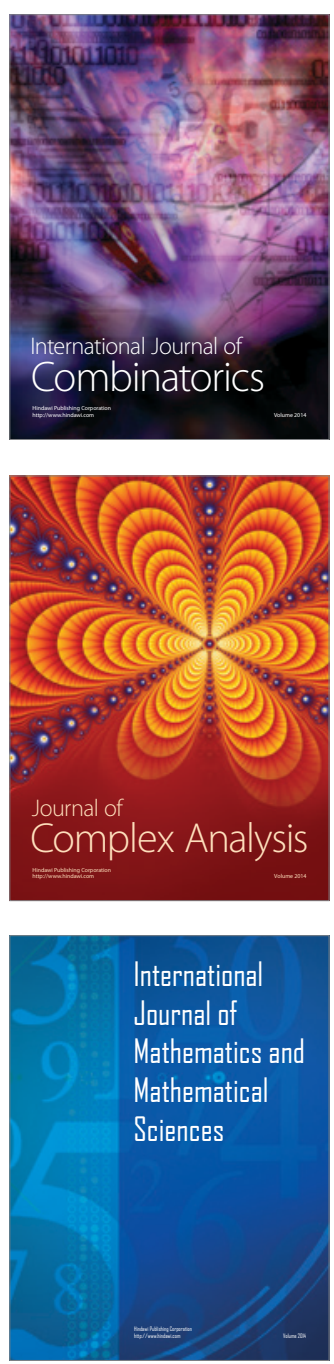
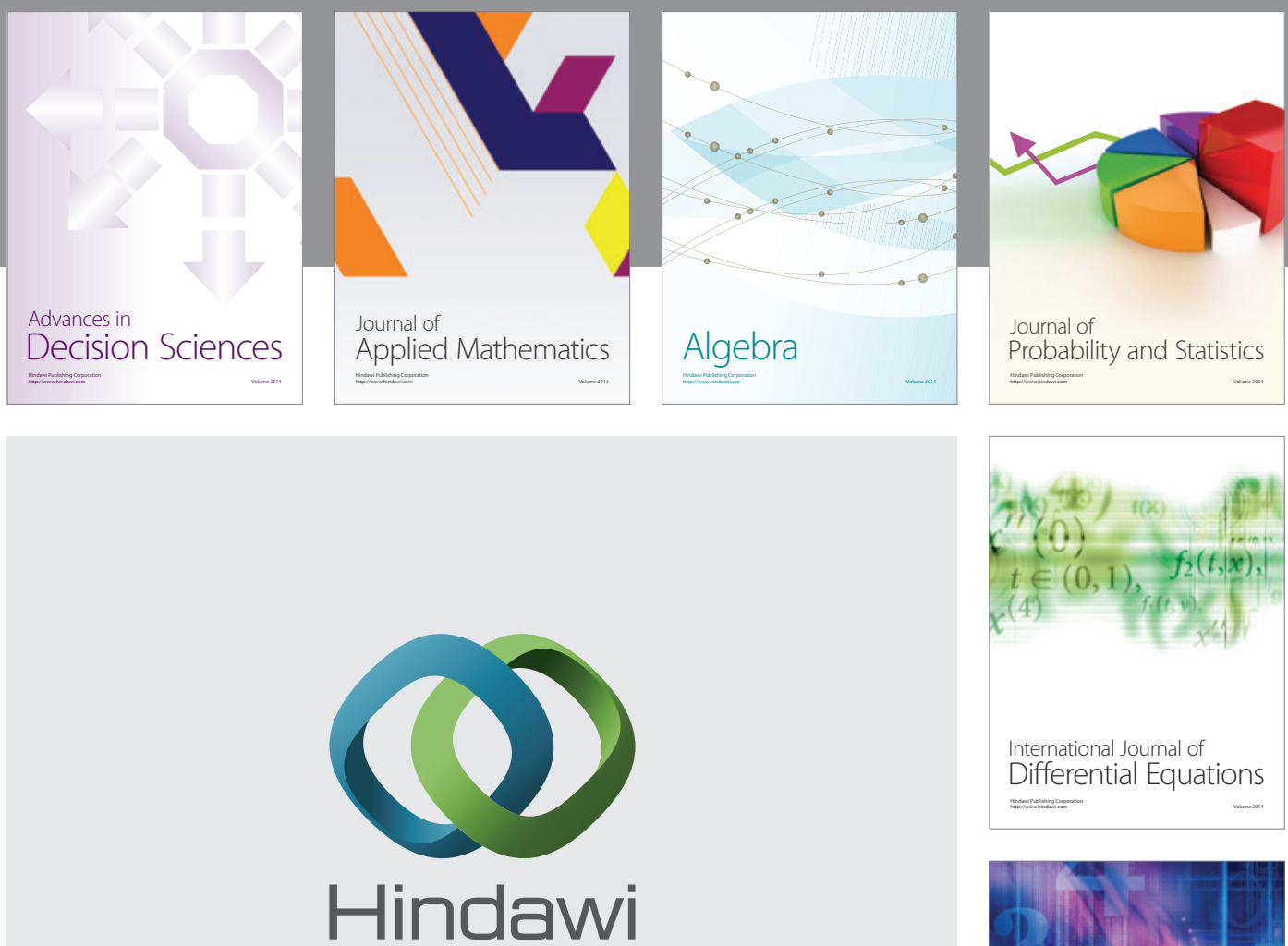

Submit your manuscripts at http://www.hindawi.com
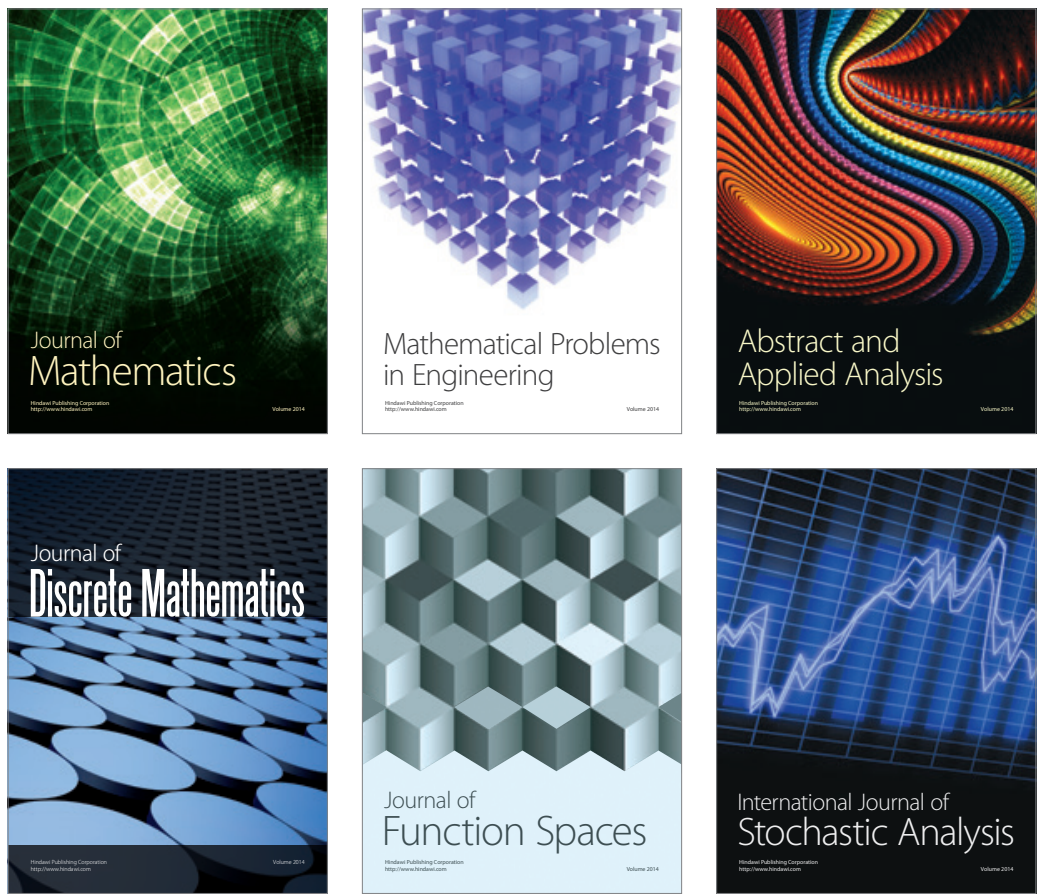

Journal of

Function Spaces

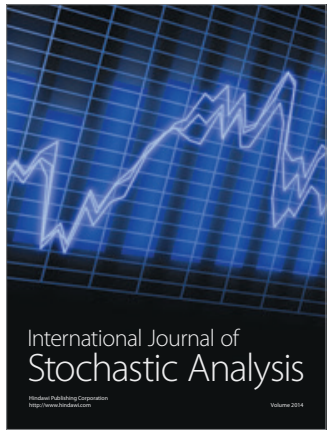

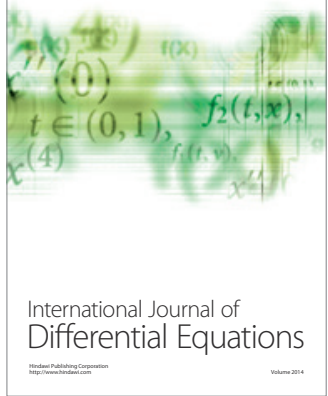
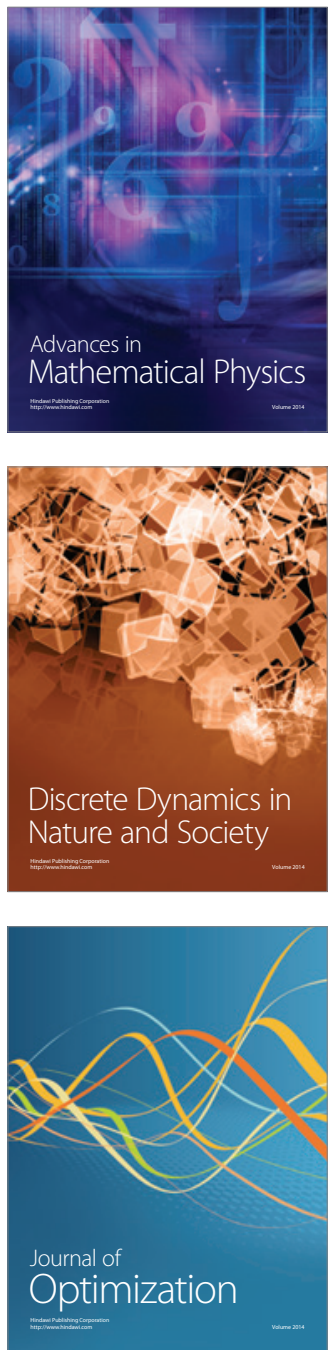